\title{
Caracterização biométrica externa, avaliação corpórea e caracterização histológica do trato gastrintestinal de gaviões-carijó (Rupornis magnirostris) apreendidos pelo CETAS/IBAMA na Paraíba
}

\author{
Millena de Oliveira Firmino ${ }^{1}$ \\ Raul Antunes Silva Siqueira ${ }^{1}$ \\ Arthur Cássio de Lima Luna ${ }^{2}$ \\ Tarsila Almeida Cavalcante ${ }^{3}$ \\ Ricardo Romão Guerra ${ }^{4 *}$

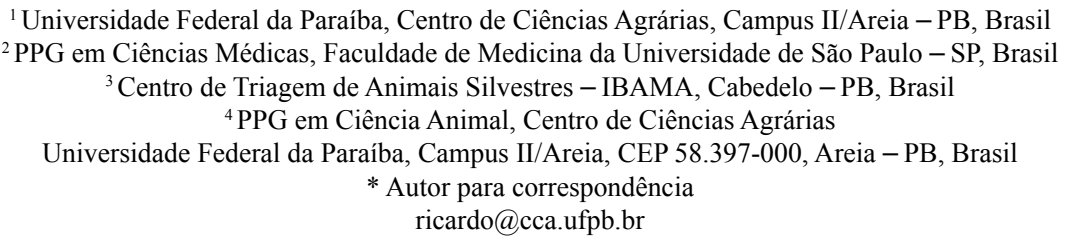

Submetido em 20/03/2013

Aceito para publicação em 21/10/2013

\section{Resumo}

Este estudo teve por objetivo analisar as medidas biométricas externas e do sistema digestório e, também, as condições corpóreas e de plumagem de gaviões-carijó apreendidos pelo CETAS/IBAMA na Paraíba, e descrever a histologia das vísceras do trato gastrintestinal (TGI), tendo como foco fornecer subsídios para novos estudos na área de nutrição animal e classificação taxonômica, bem como para sistemas de manejo e de conservação. Os espécimes foram analisados, tiveram as medidas mensuradas e coletadas amostras biológicas para procedimentos histológicos. Constatou-se que existe relação entre a condição corpórea e a perda de plumagem, demonstrando que a morfologia do TGI é semelhante à da maioria das aves já descritas, incluindo outras espécies de Accipitridae. Os resultados proporcionam subsídios para estudos posteriores envolvendo manejo nutricional, conservacionista, clínico e cirúrgico para a espécie.

Palavras-chave: Ave de rapina; Conservação; Morfologia

\section{Abstract}

External biometric characterization, body assessment, and histological characterization of the gastrointestinal tract in roadside hawks (Rupornis magnirostris) taken by CETAS/IBAMA, in Paraíba, Brazil. This study aimed to analyze measures of the external biometry and the digestive system and, also, the body and feather conditions of roadside hawks taken by CETAS/IBAMA in Paraíba, Brazil, and describe the histology of viscera in the gastrointestinal tract (GIT), focusing on the provision of means for further studies in the 
area of animal nutrition and taxonomic classification, as well as for management and conservation systems. The specimens were analyzed, measured, and biological samples were collected to undergo histological procedures. We found out that there is a relation between body conditions and feather loss, demonstrating that the GIT morphology is similar to that of most birds already described, including other Accipitridae species. The results provide means for further studies involving nutritional, conservationist, clinical, and surgical management for this species.

Key words: Bird of prey; Conservation; Morphology

\section{Introdução}

O gavião-carijó (Rupornis magnirostris, Gmelin, 1788) é pertencente ao gênero Accipitriformes e a família Accipitridae (CBRO, 2010), podendo ser encontrado desde o México até a Argentina e em todo o Brasil (FERGUSON-LEES; CHRISTIE, 2001), sendo conhecido também como indaié, gavião-pegapinto e gavião-indaié. Tem aproximadamente $36 \mathrm{~cm}$ de comprimento, possui dorso escuro e peito estriado, já a cauda tem 4-5 faixas claras em contraste às faixas cinza escuro ou negras (SICK, 1997).

Sua alimentação consiste principalmente de artrópodes, pequenos lagartos, cobras, pássaros e roedores, podendo em certas ocasiões capturar morcegos em seus pousos diurnos (SICK, 1997), portanto desenvolve um papel importante na natureza, por ser predador de topo. O mesmo contribui para a regulação das populações de pequenos roedores, além de atuar como bio-indicadores de alterações ambientais e/ou da qualidade do hábitat, devido à sensibilidade a metais pesados (BEGON et al., 1996; BILDSTEIN et al., 1998). Possui hábitos diurnos e habitam campos abertos, bordas de matas, capoeiras, margens de rios, lagos e áreas urbanizadas (SICK, 1997).

É uma das aves de rapina mais bem adaptadas às ações antrópicas, podendo ser facilmente encontrada habitando os centros urbanos (MENQ, 2011), sendo sua criação em cativeiro de interesse principalmente no Nordeste do Brasil para a prática da falcoaria (SICK, 1997). Levantamento feito no Centro de Triagem de Animais Silvestres (CETAS) $\square$ IBAMA/ PB realizado por Pagano et al. (2009), mostrou que 24 animais foram aprendidos no período de agosto de 2006 a julho de 2007 , correspondendo a $1,05 \%$ dos animais aprendidos.
Além de traçar as características externas, as medidas biométricas de uma espécie contribuem para traçar o padrão característico da espécie ou mesmo das subespécies, assim como relacionar os aspectos evolutivos, fisiológicos, taxonômicos e ecológicos da espécie (DUNNING, 1993).

Sendo assim, o presente trabalho visou avaliar a biometria externa, as condições corpóreas e a condição de plumagem que esses animais apresentavam ao chegar ao CETAS/IBAMA/PB após apreensões, e analisar por meio de mensurações e técnicas histológicas, as vísceras que compõem o TGI dos gaviões carijó, a fim de fornecer subsídios para implantar estratégias de alimentação, conservação e fornecer dados biológicos para a realização de futuros trabalhos com outras espécies.

\section{Material e Métodos}

\section{Animais}

O trabalho foi realizado no Laboratório de Histologia do Programa de Pós-Graduação em Ciência Animal da UFPB. Foram utilizados cinco espécimes de Rupornis magnirostris, sendo quatro machos e uma fêmea, que vieram a óbito por motivos não relatados, provenientes de apreensões realizadas em 2010 pelo CETAS/IBAMA $\square$ Cabedelo, PB, Brasil. O trabalho foi executado em convênio com o IBAMA sob número 02019.00129/2009-12. As carcaças foram acondicionadas sob refrigeração.

\section{Biometria}

Os espécimes foram pesados utilizando balança analítica Marte ${ }^{\circledR}$. Para a mensuração, foi utilizado paquímetro digital Caliper ${ }^{\circledR}$, as medidas externas 
analisadas foram: comprimento total com penas, comprimento total sem penas, comprimento da cauda, comprimento dos tarsos, comprimento das asas com penas, comprimento das asas sem penas, comprimento das asas fechadas, comprimento da cabeça, largura da cabeça, altura do bico, largura do bico, comprimento do bico, comprimento do dedo médio com unha, comprimento do dedo médio sem unha, largura do tórax, largura do abdômen; e as medidas do TGI foram: comprimento do esôfago, comprimento do pro-ventrículo, comprimento da moela, largura da moela, espessura da moela, comprimento do intestino e somatório de toda extensão do TGI.

\section{Condição corpórea}

As condições corpóreas em que os animais se encontravam após as apreensões foram classificadas em Boa, Magra, Caquética e Obesa, segundo a avaliação da exposição esquelética, massa muscular e quantidade de tecido adiposo no dorso e demais extensões subcutâneas (SANCHEZ, 2008) e a observação da perda ou não de plumagem e da ocorrência ou não de ferimentos.

\section{Estudo histológico do TGI}

As amostras biológicas de língua, esôfago, inglúvio, pró-ventrículo, ventrículo e intestino foram identificadas e fixadas em Metacarn (HELENO et al., 2011). Os materiais foram incluídos em parafina e cortes de $5 \mu \mathrm{m}$ foram realizados em micrótomo Leica ${ }^{C}$. Foram utilizadas as colorações histológicas de hematoxilina-eosina e tricômio de Masson. As lâminas foram analisadas em microscópio óptico e realizado o registro fotográfico com captura de imagens digitais em microscópio Olympus BX-60 e câmera Zeiss AxioCam acoplada com programa de captura de imagens digitais Ks-400.

\section{Resultados}

Os animais em estudo foram caracterizados como Rupornis magnirostirs e apresentaram peso de $155,56 \pm 41,53 \mathrm{~g}$. Os dados biométricos externos e do TGI estão detalhados nas Tabelas 1 e 2, respectivamente.

A análise das condições corpóreas mostrou que $20 \%$ dos animais tinham condição corpórea Boa (peso $201,80 \mathrm{~g}$ ) não apresentando perda da plumagem e $80 \%$ foram classificados como Magros (peso: 144,0 $\pm 37,53 \mathrm{~g}$ ) dos quais $75 \%$ dos animais tinham perda da plumagem em alguma parte do corpo (Tabela 3) (Figura 1). Dois animais apresentaram fraturas ósseas: um na asa direita e outro no membro pélvico esquerdo.

TABELA 1: Peso (g) e medidas biométricas externas (mm) de Rupornis magnirostris apreendidos pelo CETAS $\square$ IBAMA/PB em 2010. Média \pm Desvio Padrão; Máximo-Mínimo.

\begin{tabular}{|c|c|}
\hline $\begin{array}{c}\text { Medidas biométricas } \\
\text { externas }\end{array}$ & $\mathbf{m m}$ \\
\hline $\begin{array}{l}\text { Comprimento total com } \\
\text { penas }\end{array}$ & $277,10 \pm 41,78 ; 326,46-233$ \\
\hline $\begin{array}{l}\text { Comprimento total sem } \\
\text { penas }\end{array}$ & $160,13 \pm 12,06 ; 177,88-149,97$ \\
\hline Comprimento da cauda & $117,39 \pm 39,76 ; 161,37-82$ \\
\hline Comprimento dos tarsos & $.75,59 \pm 11,33 ; 84,21-56,01$ \\
\hline $\begin{array}{l}\text { Comprimento da asa com } \\
\text { penas }\end{array}$ & $272,75 \pm 45,33 ; 335,27-242,54$ \\
\hline $\begin{array}{l}\text { Comprimento da asa sem } \\
\text { penas }\end{array}$ & $131,63 \pm 23,15 ; 161,54-107,43$ \\
\hline $\begin{array}{l}\text { Comprimento das asas } \\
\text { fechadas }\end{array}$ & $192,66 \pm 28,69 ; 237,33-170,6$ \\
\hline Comprimento da cabeça & $38,43 \pm 9,71 ; 55,5-31,89$ \\
\hline Largura da cabeça & $30,73 \pm 1,67 ; 32,35-28,37$ \\
\hline Altura bico & $16,95 \pm 1,76 ; 19,78-15,18$ \\
\hline Largura do bico & $13,42 \pm 1,71 ; 15,55-11,25$ \\
\hline Comprimento bico & $24,98 \pm 3,69 ; 29,48-20,87$ \\
\hline $\begin{array}{l}\text { Comprimento do dedo } \\
\text { médio com unha }\end{array}$ & $39,07 \pm 2,88 ; 43,64-36,1$ \\
\hline $\begin{array}{l}\text { Comprimento do dedo } \\
\text { médio sem unha }\end{array}$ & $28,53 \pm 3,20 ; 25,45-33,65$ \\
\hline Largura do tórax & $37,76 \pm 2,11 ; 40,67-35,53$ \\
\hline \multirow[t]{2}{*}{ Largura do abdômen } & $31,20 \pm 4,88 ; 38,12-26,54$ \\
\hline & g \\
\hline Peso & $155,56 \pm 41,53 ; 201,8-124,35$ \\
\hline
\end{tabular}


TABELA 2: Medidas biométricas do trato gastrointestinal (TGI) (mm) de Rupornis magnirostris apreendidos pelo CETAS-IBAMA/PB. Média \pm Desvio Padrão; Máximo-Mínimo.

\begin{tabular}{lc}
\hline $\begin{array}{c}\text { Medidas biométricas do } \\
\text { TGI }\end{array}$ & mm \\
\hline Comprimento do esôfago & $70,75 \mathrm{C} \pm 12,36 ; 89,28-55,97$ \\
Comprimento do pró- & $17,35 \pm 4,06 ; 23,97-12,9$ \\
ventrículo & $35,56 \pm 8,06 ; 42,41-23,19$ \\
Comprimento moela & $18,42 \pm 4,22 ; 24,38-12,99$ \\
Largura da moela & $10,33 \pm 2,73 ; 14,65-7,18$ \\
Espessura da moela & $584,15 \pm 16,29 ; 613,06-574,91$ \\
Comprimento do Intestino & \\
Somatório do comp. do & $707,82 \pm 27,39 ; 752,08-683,49$ \\
TGI & \\
\hline
\end{tabular}

A língua do $R$. magnirostris era revestida por epitélio estratificado pavimentoso queratinizado (EEPQ). A derme era formada por papilas dérmicas, glândulas salivares e uma camada espessa de cartilagem hialina (Figura 2A). O esôfago (Figura 2B) apresentou mucosa com epitélio estratificado pavimentoso não queratinizado (EEPNQ) e lâmina própria formada de tecido conjuntivo frouxo com glãndulas mucosas. A camada muscular da mucosa era bem evidente. A camada submucosa apresentou-se delgada e foi seguida da camada muscular com dois feixes musculares; o mais interno, circular e o mais externo, longitudinal.

TABELA 3: Peso (g) de Rupornis magnirostris apreendidos pelo CETAS-IBAMA/PB em 2010 para as diferentes classificações de condições corpóreas. Peso(g) \pm Desvio Padrão.

\begin{tabular}{cc}
\hline Condição Corpórea & Peso (g) \\
\hline Boa & $201,80 \pm \mathbf{0 0 , 0 0}$ \\
Magra & $144,0 \pm \mathbf{3 7 , 5 3}$ \\
Caquética & - \\
Obesa & - \\
\hline
\end{tabular}

$\mathrm{O}$ inglúvio (Figura 2C) possuía mucosa com EEPNQ e lâmina própria com tecido conjuntivo e ausência de glândulas; e camada muscular espessa. $\mathrm{Na}$ sequência foi observada a camada submucosa e camada muscular com um feixe longitudinal.

O epitélio do pró-ventrículo (Figura 2D) era prismático simples. A lâmina própria apresentou-se escassa com muscular da mucosa delgada. A camada submucosa era repleta de glândulas multilobulares cercadas por tecido conjuntivo. Seguiu-se a camada muscular (dois feixes) e a adventícia.

FIGURA 1: Relação condições corpóreas $x$ perda de plumagem de Rupornis magnirostris apreendidos pelo CETAS-IBAMA/PB em 2010.

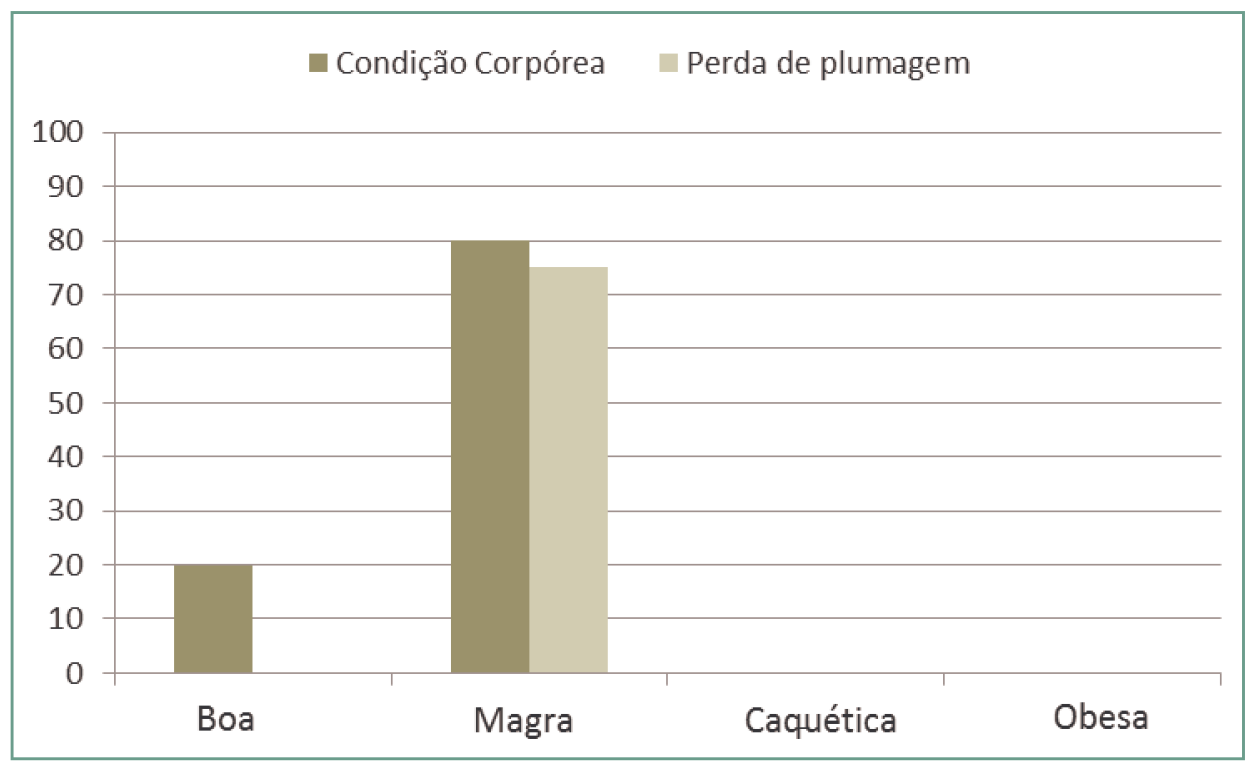

\section{Demo Version, htttp://www.verydloc.com andl htttp://www.verypdif.com}


FIGURA 2: Fotomicrografias do trato gastrointestinal de Rupornis magnirostris. A) Língua: a - epitélio estratificado pavimentoso queratinizado; b - tecido conjuntivo da derme; c - cartilagem hialina; seta - papila dérmica. Ampliação: 200X. B) Esôfago: $\mathrm{a}$ - epitélio estratificado pavimentoso não queratinizado da mucosa; $\mathrm{b}$ - lâmina própria da mucosa; $\mathrm{c}$ - camada muscular (feixes longitudinal e circular); seta - glândula da mucosa. Ampliação: 100x. C) Inglúvio: a - epitélio estratificado pavimentoso; b lâmina própria da mucosa; c - camada muscular. Ampliação: 200x. D) Pró-ventrículo: a - mucosa; b - submucosa; c - unidade glandular multilobulada; d $\square$ camada muscular (feixes longitudinal e circular); ponta seta $\square$ vaso sanguíneo da submucosa. Ampliação: 40x. E) Moela: a $\square$ cutícula; b $\square$ lâmina própria da mucosa; c $\square$ muscular da mucosa; d $\square$ submucosa e $\square$ camada muscular longitudinal; $f$ - camada muscular circular; cabeça de seta - glândula colunar da mucosa; Ampliação: 100X. F) Intestino Delgado: a - epitélio colunar simples da mucosa; b - lâmina própria da mucosa; c - submucosa; d - camada muscular (feixes longitudinal e circular); e $\square$ serosa. Ampliação: 100x. G) Intestino Grosso: a $\square$ vilos da mucosa; b $\square$ lâmina própria; c $\square$ camada muscular (feixes longitudinal e circular); d-serosa. Ampliação: 100x. A, C, D, E, F, G) Coloração de hematoxilina-eosina. B) Coloração de tricômio de Masson.
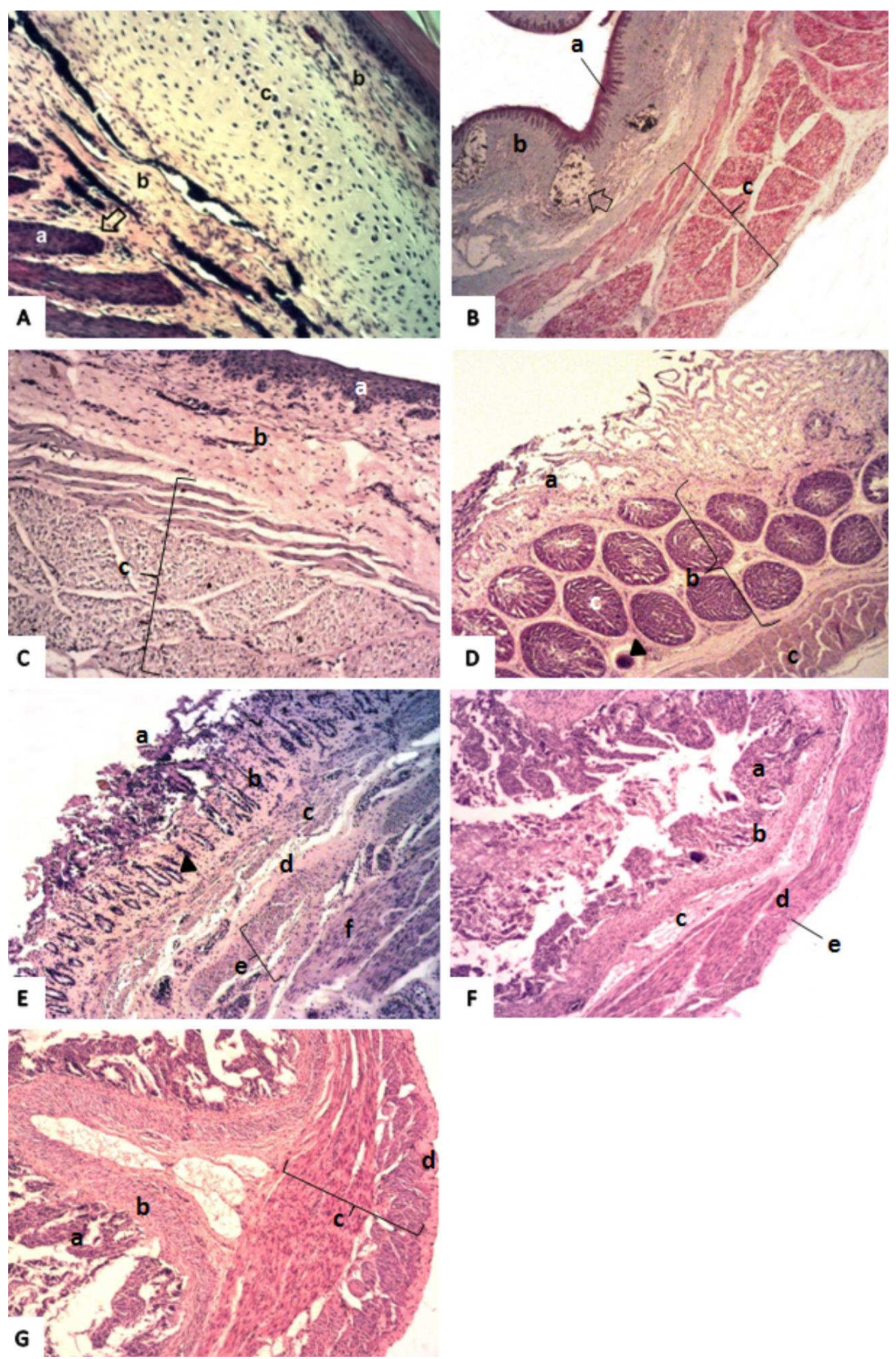
$\mathrm{O}$ ventrículo ou moela (Figura 2E) possuía uma camada cuticular quitinosa que revestia a mucosa. A lâmina própria era formada por espessa camada de glândulas tubulares com, tecido conjuntivo adjacente, seguida por camada muscular da mucosa evidente; a submucosa era esparsa e a camada muscular espessa com dois feixes: longitudinal e circular.

A porção inicial do intestino (Figura $2 \mathrm{~F}$ ) e a porção final (Figura $2 \mathrm{G}$ ) possuía mucosa com epitélio prismático simples e lâmina própria, seguida por submucosa e camada muscular dividida em feixe circular e longitudinal. Na porção final foi observado que a mucosa possuia vilos e dobras revestindo-a. A submucosa era delgada e a muscular formada por dois feixes (circular e longitudinal) bem espessa. A serosa era a última camada histológica.

\section{Discussão}

Apesar de ser uma espécie comum nas regiões metropolitanas, são escassos estudos que descrevam sua biometria externa e inexistentes os que descrevam a histologia da espécie. De acordo com as características externas os animais foram caracterizados como $R$. magnirostirs (SICK, 1997; FERGUSON-LEES; CHRISTIE, 2001). Os mesmos apresentaram peso menor que espécies da família Accipitridae, como o gavião-tesoura (Elanoides forficatus) com $407 \mathrm{~g}$ e o sauveiro-do-norte (Ictinia mississippiensis) com $280 \mathrm{~g}$ (MENQ, 2012).

Bugoni et al. (2002), em estudo no RS-Brasil com gavião-carijó, descrevem algumas medidas biométricas maiores que as encontradas em nosso estudo, são elas: comprimento de cauda (160 $\mathrm{mm} \times 117,39 \pm 39,76 \mathrm{~mm}) \mathrm{e}$ bico (28 mm vs 24,98 $\pm 3,69 \mathrm{~mm}$ ). Dessa forma, podemos supor que os espécimes encontrados na caatinga são menores que os encontrados em outras regiões do Brasil.

O comprimento do esôfago do gavião-carijó foi maior que o encontrado em carcará (Polyborus plancus) $(60 \pm 0,75 \mathrm{~mm}$ : machos e $60 \pm 1,31 \mathrm{~mm}$ : fêmeas $)$ (FRANZO, 2009), embora o mesmo seja maior e mais pesado. O pró-ventrículo, como já esperado, é menor do que em galinhas (Gallus gallus domesticus) os quais tem
$50 \mathrm{~mm}$ (MCLEOD et al., 1964). As biometrias do TGI também são de difícil comparação, visto a ausência de referências para Accipitriformes.

Segundo Turk (1982), o comprimento do trato entérico é maior em aves herbívoras e menores nas carnívoras, pois os carnívoros têm em sua dieta elementos de maior valor nutritivo e de fácil digestão requerendo um aparelho digestório simples (DYCE et al., 2010). Sendo assim, é de se esperar que o TGI de $R$. magnirostris $(707,82 \pm 27,39 \mathrm{~mm})$ seja relativamente menor do que em outras aves herbívoras como o pato doméstico (Anas platyrhynchos) (1.550-2.330 mm) e o ganso (Anser anser) (2.500-3.650 mm) (PILZ, 1937).

Sanchez (2008) em seu levantamento demonstra que $47,62 \%$ das mortes de aves traficadas advêm de distúrbios nutricionais, corroborando com nossos resultados que revelam a relação entre a má condição corporal com a perda da plumagem. Além disso, encontramos traumas ósseos advindos provavelmente dos maus tratos que esses animais são submetidos durante a captura e acondicionamento. Demonstrando, dessa forma, a necessidade de se criar instalações específicas em Centros de Triagens de Animais Selvagens que levem a recuperação dos animais para futuros programas de soltura, uma vez que as mesmas são padronizadas para todo o país.

A partir das análises histológicas foi possível observar que o epitélio da língua do $R$. magnirostris é semelhante ao encontrado no carcará ( $P$. plancus) (VIANA et al., 2011), inclusive com profundas papilas dérmicas; interdigitações entre epiderme e derme com função de aumentar aderência (BACHA; BACHA, 2003). Consonante também com de G. gallus domesticus (BACHA; BACHA, 2003).

A histologia do esôfago também estava em consonância com demais aves (BACHA; BACHA, 2003; DYCE et al., 2010), com glândulas na lâmina própria da mucosa que tem como função a produção de muco, que auxilia na passagem do bolo alimentar (JUNQUEIRA; CARNEIRO, 2004). O esôfago foi caracterizado pela evidente camada muscular da mucosa, similar a de avestruzes (Struthio camelus) jovens (MONTEIRO et al., 2009). A camada muscular, que apresentou dois 
feixes, divergiu da tartaruga-verde (Chelonia mydas) que só apresenta a camada muscular longitudinal em sua estratificação (MAGALHÃES et al., 2010).

O inglúvio, divertículo com função de armazenamento temporário de alimento (BACHA; BACHA, 2003; DYCE et al., 2010), apresentou na lâmina própria ausência de glândulas, diferentemente de perdizes (Rhynchotus rufescens) que apresentam apenas redução das glândulas deste segmento em relação ao esôfago (ROSSI et al., 2006).

O pró-ventrículo, histologicamente, estava em consonância com o de galinhas (AITKEN, 1958; SAMUELSON, 2007), avestruzes (MONTEIRO et al., 2009) e de demais aves domésticas (BACHA; BACHA, 2003).

Histologicamente o intestino do gavião-carijó possuía mucosa formada por vilosidades por toda sua extensão semelhante a outras aves (BACHA; BACHA, 2003). Tais vilosidades têm a função de aumentar a área de contato com o alimento a ser absorvido (JUNQUEIRA; CARNEIRO, 2004). Em mamíferos as vilosidades só são encontradas no intestino delgado (JUNQUEIRO; CARNEIRO, 2004; SAMUELSON, 2007), porção intestinal anterior ao ceco. O gaviãocarijó não apresentou ceco à semelhança de outros Accipitriformes e diferentemente de galinhas (BACHA; BACHA, 2003).

O presente estudo traz pela primeira vez a descrição biométrica completa do gavião-carijó, assim como a descrição histológica do seu TGI e a condição a que esses animais chegam aos CETAS após apreensões, sendo esse um dos únicos trabalhos para espécies de aves silvestres. Os resultados obtidos servirão de subsídios para futuros estudos envolvendo adequado manejo nutricional, clínico, cirúrgico e conservacionista, além de auxiliar em projetos de soltura realizados pelos CETAS.

\section{Referências}

AITKEN, R. N. C. A histochemical study of the stomach and intestine of the chicken. Journal of Anatomy, London, v. 92, n. 3, p. 453-466, 1958.

BACHA, W. J.; BACHA, L. M. Atlas colorido de histologia veterinária. 2. ed. São Paulo: Roca, 2003. 457 p.
BEGON, M.; HARPER, J. L.; TOWNSEND, C. R. Ecology: individuals, populations and communities. 3. ed. York: Blackwell Science Ltd., 1996. 1068 p.

BILDSTEIN, K. L.; SCHELSKY, W.; ZALLES, J. Conservation status of tropical raptors. Journal of Raptor Research, Albuquerque, v. 32, p. 3-18, 1998.

BUGONI, L.; MOHR, L. V.; SCHERER, A.; EFE, M. A.; SCHERER, S. B. Biometry, moltand brood patch parameters of birds in southern Brazil. Ararajuba, Londrina, v. 10, n. 1, p. 85-94, 2002.

CBRO - COMITE BRASILEIRO DE REGISTROS ORNITOLÓGICOS. Listas das aves do Brasil. 9. ed. 2010. Disponível em: <http://www.cbro.org.br>. Acesso em: 29 maio 2012.

DUNNING, J. B. Jr. Handbook of avian body masses. Boca Raton: CRC Press Inc., 1993. 371 p.

DYCE, K. M.; SACK, W. O.; WENSING, C. J. C. Tratado de anatomia veterinária. 4. ed. Rio de Janeiro: Elsevier, 2010. 872 p. FERGUSON-LEES, J.; CHRISTIE, D. A. Raptors of the world. Boston, New York: Houghton Mifflin Company, 2001. 992 p.

FRANZO, V. S. Estudo biométrico do esôfago do carcará (Polyborus plancus, Miller, 1777). Revista Científica Eletrônica de Medicina Veterinária, Garça, v. 7, n. 13, s/paginação, 2009.

HELENO, R. A.; SANTOS, L. M.; MIGLINO, M. A.; PERES, J. A.; GUERRA, R. R. Biometria, histologia e morfometria do sistema digestório do cachorro-do-mato (Cerdocyon thous) de vida livre. Biotemas, Florianópolis, v. 24, n. 4, p. 111-119, 2011.

JUNQUEIRA, L. C.; CARNEIRO, J. Histologia Básica. 10. ed. Rio de Janeiro: Guanabara Koogan, 2004. 540 p.

MAGALHÃES, M. S.; FREITAS, M. L.; SILVA, N. B.; MOURA, C. E. B. Morfologia do tubo digestório da tartaruga verde (Chelonia mydas). Pesquisa Veterinária Brasileira, Seropédica, v. 30, n. 3, p. 676-684, 2010.

MCLEOD W. M.; TROTTER D. M.; LUMB J. W. Avian anatomy. Minneapolis: Burgess Publishing Company, 1964. 327 p.

MENQ, W. Portal aves de rapina Brasil $\square$ Gavião carijó. 2011. Disponível em: <http://www.avesderapinabrasil.com/>. Acesso em: 21 jun. 2012.

MENQ, W. Portal aves de rapina Brasil $\square$ Gavião tesoura. 2012. Disponível em: <http://www.avesderapinabrasil.com/>. Acesso em: 26 dez. 2012

MONTEIRO, C. M. R.; SOUZA, N. T. M.; CARVALHO, R. G.; SOUZA, W. M. Análise histológica do trato gastrintestinal de avestruzes jovens (Struthio camelus Linnaeus, 1758). Biotemas, Florianópolis, v. 22, n. 3, p. 149-155, 2009.

PAGANO, I. S. A.; SOUSA, A. E. B. A.; WAGNER, P. G. C.; RAMOS, R. T. C. Aves depositadas no Centro de Triagem de Animais Silvestres do IBAMA na Paraíba: uma amostra do tráfico de aves silvestres no estado. Ornithologia, Cabedelo, v. 3, n. 2, p. 132-144, 2009.

PILZ H. Arrmerkmale am darmkanal des haugeflügels (gans, ente, huhn, taube). Gegenbaurs Morphologisches Jahrbuch, Leipzig, v. 79, n. 1, p. 275-304, 1937.

ROSSI, J. R.; BARALDI-ARTONI, S. M.; OLIVEIRA, D.; CRUZ, C.; SAGULA, A.; PACHECO, M. R.; ARAUJO, M. L. Morphology of oesophagus and crops of the partrigde Rhynchotus rufescens 
(Tiramidae). Acta Scientiarium. Biological Sciences, Maringá, v. 28, n. 2, p. 165-168, 2006.

SAMUELSON, D. A. Tratado de histologia veterinária. Rio de Janeiro: Elsevier, 2007. 527 p.

SANCHEZ, T. C. Causas de morte em passeriformes: comparação entre aves de vida livre residentes da região metropolitana de São Paulo e aves oriundas do tráfico. 2008. 185 f. Dissertação (Mestrado em Patologia Experimental e Comparada) $\square$ Universidade de São Paulo, São Paulo, 2008.

SICK, H. Ornitologia brasileira. Rio de Janeiro: Nova Fronteira, 1997. $912 \mathrm{p}$.
TURK D. E. The anatomy of the avian digestive tract as related to feed utilization. Journal Poultry Science, Savoy, v. 61, n. 7, p. 1225-1244, 1982.

VIANA, A. C. P.; RAMOS, A. T.; FRANZO, V. S.; VICENTIN, F. R.; VULCANI, V. A. S.; RABELO, R. E. Análise histológica da língua do carcará. In: CONGRESSO BRASILEIRO DE MEDICINA VETERINÁRIA, 38, 2011, Florianópolis. Resumos... Florianópolis: COMBRAVET, 2011. Versão eletrônica. 